Revista Ibero-Americana de Ciências Ambientais

Ibero-American Journal of Environmental Sciences

Edição Especial do Congresso de Gestão Ambiental do Baixo Amazonas

(CONGABA) Ago 2018 - v.9 - n.6

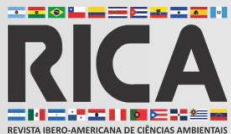

ISSN: 2179-6858

\title{
Proposta de indicadores de desenvolvimento socioeconômico e ambientalmente/ecologicamente sustentável - o IDHA e o IDHE
}

Este artigo visa propor o desenvolvimento de dois indicadores que incorporem a perspectiva sustentável em seu arcabouço metodológico, a partir da inclusão de uma dimensão ambiental/ecológica, conjuntamente às demais dimensões tradicionais do desenvolvimento humano (padrão de vida, expectativa de vida e acesso ao conhecimento), de modo a verificar a importância que essa métrica ambiental reflete nas análises de um desenvolvimento ampliado, preenchendo uma lacuna deixada pelos indicadores tradicionais, os quais não consideram a dimensão ambiental como parte integrante do instrumental analítico. A inclusão de tal dimensão é extremamente relevante na análise do desenvolvimento e sustentabilidade, já que tem um impacto direto na qualidade de vida das populações. Para tanto, propõe-se a quantificação ambiental através de dois índices: o Índice de Qualidade Ambiental e a Pegada Ecológica (Ecological Footprint), que verificam aspectos da qualidade do meio ambiente assim como aspectos biofísicos. Em seguida, incorporar-se-á tais índices ambientais às dimensões econômicas (renda per capita) e social (educação e saúde), permitindo identificar o nível de desenvolvimento humano de uma determinada localidade. Os resultados do estudo permitem analisar o nível de qualidade ambiental, assim como permitem indicar o nível de desenvolvimento humano, mostrando a essencialidade da dimensão ambiental na construção de indicadores de desenvolvimento, principalmente de caráter sustentável, podendo este apresentar-se como um fator que reduz ou aumenta o nível do desenvolvimento de uma localidade quando comparada com outras distintas, possibilitando análises comparativas pelo método de ranking ou pela estimação de impacto através de modelagem econômica-ambiental.

Palavras-chave: Desenvolvimento; Sustentabilidade; Indicadores; Ambiental; Ecológico.

\section{Proposed socioeconomic and environmentally/ecologically sustainable development indicators - IDHA and IDHE}

\begin{abstract}
This paper aims to propose the development of two indicators that incorporate the sustainable perspective in its methodological framework, from the inclusion of an environmental / ecological dimension, together with the other traditional dimensions of human development (standard of living, life expectancy and access to knowledge. ), in order to verify the importance that this environmental metric reflects in the analyzes of a broader development, filling a gap left by the traditional indicators, which do not consider the environmental dimension as an integra quality of ( allowing to identify the level of human development of a given locality. The results of the study allow us to analyze the level of environmental quality, as well as to indicate the level of human development, showing the essentiality of the environmental dimension in the construction of development indicators, especially of a sustainable nature, which may present itself as a factor that reduces or it increases the level of development of a locality when compared to different ones, allowing comparative analyzes by the ranking method or by the impact estimation through economic-environmental modeling.
\end{abstract}

Keywords: Development; Sustainability; Indicators; Environmental; Ecological.

Rhayza Alves Figueiredo de Carvalho Universidade Federal do Oeste do Pará, Brasil http://lattes.cnpq.br/2521446909903936

\section{carvalho.rhayza@gmail.com}

Abner Vilhena de Carvalho

Universidade Federal do Oeste do Pará, Brasi http://lattes.cnpq.br/8971980101556291 abner.carvalho@ufopa.edu.br

Mario Tanaka Filho

Universidade Federal do Oeste do Pará, Brasil http://lattes.cnpq.br/5710278151289710

mario.filho@ufopa.edu.br
Rodolfo Maduro Almeida (iD)

Universidade Federal do Oeste do Pará, Brasil

http://lattes.cnpq.br/1527651015984510

http://orcid.org/0000-0003-4564-825X

rodolfo.almeida@ufopa.edu.br

Jarsen Luis Castro Guimarães

Universidade Federal do Oeste do Pará, Brasil

http://lattes.cnpq.br/2403664119078137

jarsen.guimaraes@ufopa.edu.br

Márcio Júnior Benassuly Barros (iD

Universidade Federal do Oeste do Pará, Brasil http://lattes.cnpq.br/9925266223327703

http://orcid.org/0000-0001-5764-5692

marcgeo2000@yahoo.com.br
Ednéa do Nascimento Carvalho Universidade Federal do Oeste do Pará, Brasil http://lattes.cnpq.br/7125890853681726 edneancar@yahoo.com.br

André Cutrim Carvalho

Universidade Federal do Pará, Brasil andrecc83@gmail.com

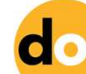

DOI: 10.6008/CBPC2179-6858.2018.006.0025
Referencing this:

CARVALHO, R. A. F.; CARVALHO, A. V.; TANAKA FILHO, M.; ALMEIDA, R. M.; GUIMARÃES, J. L. C.; BARROS, M. J. B.; CARVALHO, E. N.; CARVALHO, A. C.. Proposta de indicadores de desenvolvimento socioeconômico e ambientalmente/ecologicamente sustentável - o IDHA e o IDHE. Revista Ibero-Americana de Ciências Ambientais, v.9, n.6, p.254-272, 2018. DOI: http://doi.org/10.6008/CBPC21796858.2018 .006 .0025 


\section{INTRODUÇÃO}

O desenvolvimento deve ser entendido como um processo de transformação da sociedade relacionado a mudanças qualitativas significativas, que, em geral, acontecem de forma cumulativa. Decerto, alterações no nível de expectativa de vida estão associadas a diversas oportunidades sociais que são cruciais como serviços de saúde, desempenho educacional, liberdades políticas que fomentam uma melhor qualidade de vida para a população, mesmo dispondo de baixos níveis de renda, deste modo, a ênfase nesses serviços é válida, particularmente em economias pobres, na qual essas disposições contribuíram para o crescimento econômico (VEIGA, 2010).

Existem várias perspectivas sobre o desenvolvimento, dentre as quais consideram distintas dimensões em sua composição. No entanto, desde os anos 70, a atenção dada a problemática ambiental levou a uma ampla (re)conceituação do desenvolvimento, em termos de ecodesenvolvimento, recentemente designado como desenvolvimento sustentável, em que acrescenta a sustentabilidade ambiental à dimensão de sustentabilidade social e de viabilidade econômica, considerando um duplo imperativo ético de solidariedade com as gerações presentes e futuras, de modo que apenas as soluções que considerem impactos positivos nessas três categorias, social, ambiental e econômico, serão denominados desenvolvidas (SACHS, 2008).

Dessa forma, um dos componentes essenciais que integram o processo ampliado do desenvolvimento é a dimensão ambiental, que deve ser manejada cautelosamente, já que é de suma importância para a manutenção da vida, em que a preocupação com essas condições tem sido fundamentada pelo impacto direto na qualidade de vida dos indivíduos, pois existe uma ligação direta entre aspectos sociais e ambientais, alterações nas disponibilidades e uso imprudente da biodiversidade podem alterar as condições de vida das populações tais como modificações nos níveis de saúde, poluições de ar, águas e extinção de recursos naturais.

O desenvolvimento sustentável passa a ser descrito em cinco pilares, dentre os quais, o social, devido a heterogeneidade estrutural entre as localidades, o territorial, relacionando-se à distribuição espacial dos recursos, de atividades e população, o fator econômico que é imprescindível por conta da viabilidade econômica, o político, por conta da democracia exercer valor fundamental, além de garantir transparência e a responsabilidade necessária ao funcionamento dos processos de desenvolvimento e por fim, a questão ambiental, relacionado aos sistemas de sustentação da vida como provedores de recursos e como recipientes para a disposição de resíduos, o progresso de todas as dimensões corresponde a perspectiva sustentável (SACHS, 2008).

As variáveis que compõem a dimensão ambiental recebem destaque na atualidade, particularmente no tocante aos aspetos ligados ao desenvolvimento na sociedade, que possibilitam integrar uma visão ampliada da realidade, emergindo concomitante a necessidade de indicadores que contemplem tal questão, para designar a situação, presença, ausência e ameaças possíveis a tais recursos ligado aos aspectos 
socioeconômicos, permitindo situar a sociedade em relação ao desenvolvimento sustentável, que congrega além de dimensões tradicionais do desenvolvimento as medidas ambientais.

Alguns procedimentos estatísticos têm como escopo quantificar os fenômenos observados em uma sociedade, principalmente quando acontecem mudanças e oscilações, revelando o entendimento e interpretação sobre determinada população em um período. O uso de um conjunto de dados ocorreu principalmente, por indicadores bem tradicionais como PIB e PIB per capita considerados índices de produção agregada da economia, que surgiram devido à necessidade de mensurar o nível de renda, designando como representativo de uma característica em particular.

A quantificação na pesquisa permitir visualizar, a partir de um conjunto de dados e tratamentos específicos, alguns aspectos que não se revelam em análises primárias. Logo, existem situações em que as grandezas numéricas são essenciais para discussões de temas relevantes para a sociedade, possibilitando mensurar fenômenos em números, de acordo com critérios estabelecidos, desta forma, verificar a intensidade de um fenômeno não é algo trivial.

Portanto, o propósito deste artigo é apresentar uma contextualização em relação ao tema do Desenvolvimento Sustentável, demonstrando a importância que índices ambientais exercem para a formação de uma abordagem multidimensional, com a inclusão da dimensão ambiental por intermédio de dois indicadores ambientais conjuntamente às outras dimensões tradicionais do Índice de Desenvolvimento Humano (IDH), levando em conta os aspectos relacionados a qualidade do meio ambiente, descritos no Índice de qualidade ambiental (IQA) e a sustentabilidade, verificada pela índice da Pegada Ecológica (EF), verificando, dessa maneira, a situação ambiental de cada localidade, ou seja, se a dimensão ambiental contribui ou penaliza a localidade. Isso, levando em conta se o desenvolvimento, com suas dimensões tradicionais, somados aos Índices de Desenvolvimento Humano Ambiental (IDHA) e de Desenvolvimento Humano Ecológico (IDHE) estão cooperando para alcançar o Desenvolvimento Sustentável.

\section{METODOLOGIA}

De forma geral, podemos identificar os diferentes delineamentos e tipos de pesquisa quanto: à sua abordagem; sua natureza; seus objetivos e; seus procedimentos. "De acordo com as características da pesquisa, poderão ser escolhidas diferentes modalidades de pesquisa, sendo possível aliar o qualitativo ao quantitativo" (SILVEIRA et al., 2009).

Para tanto, o tipo de pesquisa deste ensaio tem uma abordagem considerada tanto quantitativa como qualitativa. Na qual a pesquisa quantitativa recorre à linguagem matemática para descrever as causas de um fenômeno, as relações entre variáveis, permitindo recolher mais informações do que se poderia conseguir isoladamente. No presente estudo, o uso da operacionalização de indicadores ambientais permite quantificar a qualidade e a situação do meio ambiente, e de maneira conjunta interpreta as condições do meio ambiente, por intermédio da compreensão, explicação, dos fenômenos ambientais (FONSECA, 2002).

A natureza da pesquisa é classificada como aplicada, tendo por objetivo gerar conhecimentos para aplicação prática, como a quantificação das características ambientais, no que se refere aos objetivos é 
classificada como descritiva, na qual o estudo pretende descrever a qualidade do meio ambiente, assim como o nível de desenvolvimento considerado sustentável, que também leva em consideração aspectos sociais e econômicos. Este ensaio utiliza procedimentos metodológicos que caracterizam a pesquisa como bibliográfica, já que foi realizado a partir do levantamento de referências teóricas já analisadas, e publicadas por meios escritos e eletrônicos, como livros, artigos científicos, páginas de web sites (FONSECA, 2002).

\section{Indicador, índice e medidas: definição e importância}

Usualmente, o conceito de índice e indicador se confunde, pois ambos funcionam como uma sinalização para manifestar a situação de um sistema determinado, pois são valores estáticos e em grande parte das análises superficiais designam índice e indicador igualmente, portanto são utilizados em grande parte de forma errônea, como sinônimos. Com intuito de facilitar a quantificação, o indicador é uma ferramenta que pode se referir a um dado individual ou um agregado de informações, tendo propósitos específicos (SICHE et al., 2007).

No entanto, a validação e aceitação desses instrumentos quantitativos exigem alguns requisitos essenciais para sua construção e operacionalização, já que serão representações. Segundo Mueller et al. (1997, citado por SICHE et al., 2007) “[...] um bom indicador deve conter os seguintes atributos: simples de entender; quantificação estatística e lógica coerente; e comunicar eficientemente o estado do fenômeno observado".

Para a maioria dos estudiosos, a diferença é que o índice revela o valor agregado final de todo um procedimento de cálculo e a correta interpretação da realidade de um sistema simples ou complexo (natural, econômico ou social), utilizando, em seu cálculo, bases científicas e métodos adequados, utilizando-se inclusive, indicadores como variáveis que o compõem, de modo que um índice pode se transformar num componente de outro índice. Enquanto que o termo indicador é um parâmetro selecionado e considerado isoladamente ou em combinação com outros para refletir sobre as condições do sistema em análise, e possível potencialidade pressagiadora, devido a sua metodologia considerar mudanças internas com o tempo devido alterações de agentes externos ou novos arranjos internos (SICHE et al., 2007).

Portanto, os índices e indicadores são instrumentos que podem facilitam na investigação de uma série de acontecimentos presentes e possibilitando construir trajetórias possíveis. Entretanto, o uso indiscriminado dos conceitos de índice e indicador favorece a reprodução de erros analíticos, já que podem ser utilizados como referências entre os pesquisadores.

Os indicadores permitem dentre outras coisas comparações no tempo e no espaço, avaliar as condições, tendências, além de comparar lugares e situações, Contudo, ressalta-se que podem existir dificuldades de análise, sobretudo, nos indicadores ambientais, por conta de limitações das variáveis (com necessidade de adequação) e pelo desconhecimento de algumas funções ecossistêmicas dos recursos naturais, o que não inviabiliza a construção dos indicadores, mas demonstrando suas limitações. Portanto, a ausência de indicadores universais se deve principalmente pela variação de problemas ou do objetivo da 
análise, ressaltando ser importante a construção destes indicadores, levando em conta as particularidades da realidade analisada (SOUZA, 2004; MOURA et al., 2005).

Siche et al. (2007) salienta que "[...] um índice é um dado mais apurado que provém da agregação de um jogo de indicadores ou variáveis e que pode interpretar a realidade de um sistema. Por outro lado, um indicador normalmente é utilizado como um pré-tratamento aos dados originais". Dessa forma, um índice pertence a uma escala considerada mais elevada que o indicador, ou seja, um conjunto de indicadores agregados ou ponderados.

É importante frisar que o indicador também é um instrumento dinâmico, já que mudanças de conceitos, em escalas temporais e locais também são acompanhadas por alterações nos indicadores propostos para representar determinado processo. Com o intuito de compreender a realidade, os indicadores descrevem uma faceta da mesma, levando em conta a escolha de qual aspecto da realidade será tratado e a definição do conceito abstrato que será utilizado para explicação do fenômeno, assim, definições são as bases para qualquer medida (SOLIGO, 2012).

Ressaltando que há várias definições sobre o que é um indicador, podendo este ser um instrumental quantitativo ou qualitativo, não sendo restrito a apenas uma daquelas esferas, representando, em sua essência, uma variável que assume um valor em um tempo específico. Por sua vez, uma variável é uma representação de um atributo de um determinado sistema, incluindo qualidade, característica e propriedade (QUIROGA, 2001).

Em uma visão mais abrangente, um indicador é um sinal que aponta uma determinada condição, com a finalidade de comunicar sobre informações oportunas e auxiliar na tomada de decisões (GALLOPÍN, 1996, 2003). Dessa maneira, um indicador não é apenas uma estatística, tem como objetivo apontar sobre a existência de riscos, potencialidades e tendências no desenvolvimento de um determinado território para que, em conjunto com a comunidade, decisões possam ser tomadas de forma mais racional (GUIMARÃES, 1998).

Para a construção de um índice, todos os indicadores componentes são transformados previamente em índices, que variam entre 0 e 1. A transformação do indicador é feita através de uma fórmula matemática simples, conforme a seguinte equação: Índice=valor observado - valor mínimo/valor máximo - valor mínimo. A expressão acima transmite uma ideia também muito simples, que é de uma relação entre as distâncias do valor observado em relação ao pior(es) e ao melhor(es) valores definidos para ele, ou seja, quanto mais o valor observado para este componente se aproxima do mínimo (ou do pior valor), menor é o numerador da equação e mais próximo de 0 o índice da localidade vai estar. A escolha dos valores de máximo e mínimo é importante, pois impacta diretamente no valor a ser obtido para o índice, impactando, também, na dispersão dos valores do índice entre as diversas localidades.

Algumas variáveis são utilizadas para representar algumas dimensões (sociais, ambientais, políticas e econômicas) que compõem o desenvolvimento, já que envolve uma conceituação dinâmica, e assim, usada como referência para construção de indicadores. A exposição dos valores de determinado conteúdo tem um conjunto de implicações, que vão desde de alarme até punições. Além disso, existem outras nomenclaturas 
específicas utilizadas nos métodos quantitativos referentes a variáveis analisadas, um exemplo é o PIB per capita, que durante muito tempo foi utilizado como proxy de nível desenvolvimento socioeconômico pelo mundo. De acordo com Sandroni (2007) proxy “[...]significa a variável utilizada na análise de regressão para substituir outra teoricamente mais satisfatória, nos casos em que não se dispõe de dados para esta última ou não é possível obtê-los[...]".

As diferentes áreas do conhecimento têm se empenhado para construir modelos que representem uma simplificação da realidade, bem como a reprodução de uma relação de antecedentes (causas) e consequentes (efeitos) e a estes serem determinísticos, na qual a causa e efeito estão ligadas diretamente, ou estocásticos vinculados indiretamente. Esses aspectos relevantes possibilitam quantificar e associar entre grandezas, por meio do estabelecimento e refinamento, a capacidade de medição em termos de unidades e instrumentos apropriados, contudo não é tarefa fácil (TRZESNIAK, 1998).

\section{Os indicadores do desenvolvimento sustentável e a construção da métrica da dimensão ambiental}

Na perspectiva de sustentabilidade, o escopo é caracterizar a interação entre a atividade antrópica e o meio ambiente, bem como trabalhar um conceito que é complexo, dinâmico e diverso. Desta forma, o maior desafio da sustentabilidade, é a quantificação, de modo a efetuar indicadores ambientais para produzir um retrato, ou um conjunto de sinais que facilitam a avaliação do progresso de uma determinada região na busca pelo desenvolvimento sustentável, sendo ferramentas crucias no processo de identificação de problemas, reconhecimento dos mesmos, formulação, implementação e avaliação de políticas.

Nesse sentido, a nova dimensão refere-se à sustentabilidade ambiental, acrescida à sustentabilidade social, fundamenta-se em uma solidariedade tanto sincrônica quanto diacrônica com as gerações atuais e futuras, respectivamente. Deste modo, a abordagem que envolve uma relação direta entre essas sustentabilidades e a viabilidade econômica, é denominada inicialmente como ecodesenvolvimento nos anos 70, e depois desenvolvimento sustentável, logo quando este tripé apresenta impactos positivos em ambos os elementos, social e ambiental se caracteriza como desenvolvido (SACHS, 2008).

Na operacionalização deste conceito emerge, nas agendas de governos e da sociedade, a necessidade de pensar em novas formas de mensurar o crescimento e de garantir a existência de um processo transparente e participativo para o debate e para a tomada de decisões em busca do desenvolvimento sustentável. Neste contexto, um conjunto de indicadores de sustentabilidade exerce a função de advertir à comunidade sobre riscos e tendências do desenvolvimento, atendendo à necessidade de mensurar a distância entre a situação atual de dada sociedade e seus objetivos de desenvolvimento sustentável, se constituindo uma carta de navegação sobre o futuro, onde se vislumbra um destino, se acompanha o trajeto e se corrigem os rumos (GUIMARÃES, 1998; GUIMARÃES et al., 2009).

No decorrer de três décadas, desde a Conferência das Nações Unidas sobre meio ambiente em 1972 até a cúpula sobre desenvolvimento sustentável, realizada em Joanesburgo, em 2002, o conceito de desenvolvimento sustentável vem sendo ajustado, pois a preocupação com os aspectos ambientais passaram a ser incorporadas nas principais discussões no globo, almejando mudanças de comportamento na forma 
como os seres humanos se relacionam com o meio ambiente, bem como no modo de formular, implementar e avaliar políticas públicas de desenvolvimento. O relatório de Brundtland (1986) difundiu o conceito de desenvolvimento sustentável, em que pretendiam sustentar ou aumentar concomitantemente o meio ecológico por meio da qualidade ambiental e estoque de recursos, com as duas dimensões clássicas de bem estar econômico e a justiça social (SACHS, 2008; GUIMARÃES et al., 2009; ENRÍQUEZ, 2010).

Em síntese, para que indicadores sejam instrumentos de um processo de mudança rumo ao conceito de desenvolvimento sustentável, eles devem congregar características que permitam: mensurar diferentes dimensões de forma a apreender a complexidade dos fenômenos sociais; possibilitar a participação da sociedade no processo de definição do desenvolvimento; comunicar tendências, subsidiando o processo de tomada de decisões; e relacionar variáveis, já que a realidade não é linear nem unidimensional (GUIMARÃES et al., 2009).

Denominados de índices de segunda e terceira geração os quais incorporam dimensões básicas do desenvolvimento como: renda, saúde e educação, com o objetivo de monitorar o desempenho desenvolvimentista por tais indicadores essenciais do fenômeno. Assim, é exatamente o problema que pretende ser superado com essa abordagem considerada como quarta geração, denominados assim, pois incluem outras dimensões importantes do desenvolvimento, como por exemplo, a ambiental (VEIGA, 2010).

O desenvolvimento, em sua perspectiva sustentável, por meio da inclusão da dimensão ambiental possibilita verificar como tal métrica afeta o nível de desenvolvimento de cada localidade, revelando sua classificação com relação ao seu nível de desenvolvimento e, assim analisar o tipo de padrão de crescimento que cada local vem adotando.

Com o intuito de quantificar as principais dimensões do desenvolvimento sustentável, surge duas propostas de adaptações metodológicas, considerando em conta aspectos socioeconômicos e ambientais, denominadas de Índice de Desenvolvimento Humano Ambiental (IDHA) e Índice de Desenvolvimento Humano Ecológico (IDHE), na qual o primeiro passo consiste na criação de um subíndice que representa a dimensão ambiental para os tais índices conhecidos como Índice de Qualidade Ambiental (IQA) e Pegada Ecológica (EF), em seguida, esse subíndice será incluído ao cálculo do IDHM tradicional de maneira distinta, ou seja, dois índices com quatro dimensões, levando em conta as dimensões expectativa de vida, acesso à educação e padrão de vida.

O intuito daqueles indicadores com a dimensão ambiental é de verificar de duas formas distintas, qual(is) o(s) impacto(s) ambientais com relação ao desenvolvimento, de forma a suprir a inconsistência do IDHM, no que tange a inclusão da variável ambiental (sustentabilidade) na estrutura de um indicador consagrado.

\section{Os subíndices dos indicadores ambientais}

Visando incorporar a dimensão ambiental na composição de um índice geral de desenvolvimento (econômico, social, e ambiental), seja de cunho internacional/nacional, IDH, ou seja na esfera municipal, 
IDHM, é de suma importância o cálculo de dois subíndices, de dimensão puramente ambiental: o Índice de Qualidade Ambiental (IQA); e a Pegada Ecológica (EF).

Em que as dimensões ambientais irão incorporar-se as dimensões tradicionais do IDH pelo Índice de Qualidade Ambiental (IQA) e/ou pela Pegada Ecológica (EF), envolvendo a questão da sustentabilidade, assim possibilitando o entendimento amplo de desenvolvimento conjuntamente aos fatores socioeconômicos, e, ambientais, tanto na qualidade desse ambiente quanto nas questões biofísicas. Portanto a integração das duas dimensões - socioeconômica e ambiental - busca suprir as deficiências de ambos por meio de sua complementariedade, parecendo ser uma alternativa lógica plausível, formando uma quarta dimensão no Índice, passando a compor novos índices: o IDHA e IDHE.

\section{O Índice de Qualidade Ambiental (IQA)}

Algumas pesquisas realizam adaptações metodológicas quanto a formulação de uma dimensão ambiental e na possibilidade de tentar agregar aos indicadores socioeconômicos medidas que considerassem o comportamento ambiental, dessa forma, para a proposta sugerida foram utilizadas as sugestões de Silva et al. (2004) e Oliveira (2013), utilizados para a verificar a dimensão ambiental através do cálculo do Índice de Qualidade Ambiental (IQA) e Pegada Ecológica (EF), respectivamente nos países, foi construído a partir de tais condicionantes, como a impossibilidade de quantificar estritamente a qualidade ambiental, atribuindo a seu cálculo ponderadores diferenciadas.

Para o cálculo do IQA, considerou-se a preocupação ambiental de cada país com um ponderação de $1 / 2$, julgando ser de maior importância tal componente, utilizando como proxies o índice de área protegida (IA2), com ponderação de $1 / 3$ ao índice de cobertura por florestas (IA1), refletindo o patrimônio natural que o país detém, e por fim, atribui-se $1 / 3$ de ponderação ao índice de eficiência da energia (IA3) na participação do IQA.

O IQA é composto por índices da dimensão ambiental, e seu cálculo e obtido por uma média ponderada que contempla três subdimensões daquela variável, sendo um indicador sintético que tenta congregar num único valor quantitativo, uma dimensão normativa e qualitativa de como é o estoque qualidade ambiental, conforme descrição apresentada no quadro 1.

Quadro 1: Componentes da dimensão ambiental do IQA.

\begin{tabular}{|c|c|c|c|}
\hline ÍNDICE & \multicolumn{3}{|c|}{ Proxies dos Índices de Qualidade Ambiental } \\
\hline Subíndices do IQA & $\begin{array}{c}\text { Porcentagem da área terrestre } \\
\text { coberta por florestas (CFi) }\end{array}$ & $\begin{array}{c}\text { Razão entre a área protegida e a } \\
\text { superfície terrestre e (RAPSTi) }\end{array}$ & $\begin{array}{c}\text { PIB por unidade de } \\
\text { energia utilizada (PIB/Ei) }\end{array}$ \\
\hline Fonte dos Dados dos subíndices & $\begin{array}{c}\text { (Censo Agropecuário do IBGE) } \\
\text { e (TERRACLASS) }\end{array}$ & $\begin{array}{c}\text { (TERRACLASS) } \\
\text { (PRODES) }\end{array}$ & $\begin{array}{c}\text { (Censo Demográfico do } \\
\text { IBGE) }\end{array}$ \\
\hline
\end{tabular}

Fonte: Silva et al. (2004).

A porcentagem da área terrestre coberta por florestas (CFi) reflete o estoque de recursos essenciais para manutenção da biodiversidade e qualidade ambiental, sobretudo ao nível da regeneração do ar, e além do mais verifica a paisagem como parte integrante da relação entre o homem e a natureza. Portanto, a variável mede face à superfície terrestre total, a parte desta que está coberta por florestas sendo calculado 
pelo índice de cobertura por florestas: $I C F_{i}=\frac{C F_{i}-C F_{\text {minima }}}{C F_{\text {máxima }}-C F_{\text {minima }}}$, onde $C F i$ significa a porcentagem de área terrestre coberta por florestas. A Razão entre a área protegida e a superfície terrestre (RAPST), de acordo com o Relatório de Desenvolvimento Humano da ONU, essa, Segundo Silva et al. (2004):

Refere-se às áreas protegidas total ou parcialmente, de pelo menos 1000 hectares, que foram definidas como parques nacionais, monumentos naturais, reservas naturais ou santuários de reserva de vida selvagem, paisagens e marinhas protegida, ou reservas científicas com acesso público limitado, não inclusos nos dados os sítios protegidos por lei local ou provincial.

Esta variável é de extrema importância para perceber o nível de preocupação de um país com a sua diversidade biológica. Esta subdimensão é calculada pelo índice de razão entre a área protegida e a superfície terrestre do local $i$. Tem-se IRAPST $i=\frac{R A P S T_{i}-\text { RAPST }_{\text {minima }}}{\text { RAPST máxima }^{-R_{\text {APST }} \text { mínima }}}$, onde IRAPSTi significa o razão entre a área protegida e a superfície terrestre do país $i$.

Atenta-se para o fato de um valor mais elevado do PIB por unidade de energia utilizado significa que para um mesmo nível de produto originado, é necessário consumir menos energia, logo, potencialmente são necessárias menores emissões de poluentes relativas em comparação com locais de mesmas condições, mas com um índice superior. Obviamente que, pelo contrário, a um valor da variável mais baixo, corresponderá um nível de emissões relativas superior (SILVA et al., 2004).

A priori, daqui poderão surgir importantes consequências ao nível da análise da escala municipais das economias: é de esperar que um município fortemente industrializado, necessite de grandes consumos energéticos para obtenção do seu PIB pelo que apresentará um razão penalizadora em termos de qualidade ambiental; já um município maioritariamente terciário deverá observar um valor alto do razão, na medida em que os serviços não são grandes consumidores relativos de energia; quanto aos municípios em que o dominante é o setor agrícola, sobretudo municípios em vias de desenvolvimento e portanto com estruturas pouco mecanizadas e métodos de produção pouco utilizadores de energia mas também muito pouco produtivos, o que poderá conduzir a razões baixos de PIB por unidade de energia (SILVA et al., 2004).

A subdimensão do PIB por unidade de energia utilizada será calculada pelo índice de razão Índice de eficiência da energia $i: \frac{P I B}{E_{i}}=\frac{P I B / E_{i}-P I B / E_{\text {minima }}}{P I B / E_{\text {máxima }}-P I B / E_{\text {mínima }}}$, na qual $\mathrm{PIB} / \mathrm{E}_{\mathrm{i}}$ : representa a razão entre o PIB (dólares PPC) e quilogramas de energia consumida equivalente a petróleo no local $i$.

Outros trabalhos utilizaram no cálculo desse componente a proxy, a participação do setor industrial no PIB municipal (\%), adaptando metodologicamente para a mensuração a nível municipal, de modo a verificar a poluição industrial, já que quanto maior a participação da indústria no PIB municipal, maior seria o nível de poluição nesses municípios (ARAÚJO, 2009; BARBOSA, 2009; ROSSATO 2006). Destaca-se o cuidado que deve ser dado a variável $\mathrm{PIB} / \mathrm{E}_{\mathrm{i}}$, no tocante a sua interpretação, pois depende da estrutura econômica do país, bem como da eficiência produtiva do input energia, ou seja, das unidades de utilização de energia (SILVA et al., 2004).

O PIB por unidade de energia utilizada é a energia comercial utilizada medida em quilogramas de equivalente petróleo, será verificada pelos dados do censo demográfico. Este razão fornece uma medida de 
eficiência da energia, mostrando estimativas comparáveis e consistentes do PIB real entre países relativamente aos inputs físicos (unidades de utilização de energia), contribuindo positivamente para a qualidade ambiental, já que é uma medida de eficiência na utilização de recursos energéticos (na sua maioria não renováveis e poluentes) permite-nos comparar o desempenho de localidade a este nível (SILVA et al., 2004).

Cada subdimensão do IQA contempla um peso e uma definição, conforme a equação IQA=1/3 (ICFi) $+1 / 2($ IRAPSTi $))+1 / 6$ (PIB/Ei). Vale ressaltar as limitações existentes quanto às variáveis utilizadas para construir o IQA, principalmente no que concerne à escassez de informações estatísticas disponíveis sobre o meio ambiente e a necessidade de garantir a operacionalidade e simplicidade do índice.

Dessa forma, é relevante a adaptabilidade de alternativas quanto a inclusão de novas variáveis para compor o índice, apresentando as performances dos municípios no que se refere a perspectiva sustentável, ou seja, se apresentam ou não bons desempenhos em tal abordagem (SILVA et al., 2004). A inclusão de uma variável ambiental, pode sofrer adaptações quanto a escolha de tais, partindo de questões subjetivas oriundas do pesquisador, já que não existe concordância na literatura sobre variáveis que se referem a tal métrica (SOARES et al., 1999).

\section{A Pegada Ecológica (Ecological Footprint - EF)}

De acordo com o objetivo da pesquisa, a outra possibilidade de mensurar o impacto da dimensão ambiental no processo de desenvolvimento será realizado por meio do cálculo da pegada ecológica, por se tratar de um indicador de sustentabilidade amplamente utilizado para verificar a questão ambiental (LISBOA et al., 2010; CERVI et al., 2010; FEITOSA et al., 2013; FIRMINO et al., 2009)

O índice da Pegada Ecológica (Ecological Footprint) foi realizado em 1996, por William Rees e Mathis Wackernagel que propuseram essa ferramenta como forma de medir o desenvolvimento sustentável, com o intuito de verificar a pressão exercida pelo homem, ou seja, a biocapacidade (oferta) do planeta sobre o meio ambiente, enquanto medida de (demanda), por meio da quantificação de hectares que uma pessoa necessita para produzir o que consome, ou seja, sustentar seu modo de viver. A diferença negativa entre a demanda e oferta é chamada de "overshoot", é considerado o estado que ultrapassa os limites biofísicos do planeta.

O Cálculo é feito pelo Global Footprint Network (2010) e divulgado pela World Wildlife Fund - WWF, exibindo a quantidade de hectares globais (gha) de terra e água essenciais para a manutenção da humanidade com um determinado padrão de utilização dos recursos e emissão de rejeitos (CERVI et al., 2010; OLIVEIRA, 2013).

Logo, o objetivo deste índice é estimar o tamanho da marca deixada pela humanidade no planeta, formado por várias categorias como: áreas de cultivo, pastagens, áreas urbanizadas, estoques pesqueiros, produtos florestais e carbono, que é a quantidade de floresta necessária para absorver as emissões adicionais de dióxido de carbono que os oceanos não conseguem absorver, possibilitando verificar uma série de impactos ambientais distintos, tanto no lado do input (recursos naturais) quanto do output (degradação ambiental). Isso significa que é o somatório de todas as parcelas, representando o capital natural consumido 
pela sociedade, na qual é comparada com o espaço existente no planeta, ou seja, biocapacidade, revelando os resultados da sustentabilidade na localidade (OLIVEIRA, 2013; CERVI et al., 2010).

O cálculo do índice é dado pelo somatório de todas essas dimensões, envolvendo a razão da estimativa do padrão de consumo pessoal médio de uma população per capita pela estimativa do rendimento médio do item consumido (padrão de eficiência), multiplicado por um fator de equivalência, formando um único número que possibilita a comparabilidade e instrumento comparativo entre localidades e para tomadores de decisão política (OLIVEIRA, 2013). O cálculo da Pegada Ecológica (EF) é formado pelo somatório de todas as dimensões pela equação $E F_{P}=\sum i\left(\frac{P_{i}}{Y_{w, i}}\right) \cdot E Q F_{i}$, onde: $P_{i}=$ consumo médio por pessoa do país; $Y_{w, i}=$ eficiência da gestão dos rejeitos desse consumo; e $E Q F_{i}=$ fator de equivalência (medida do padrão de consumo do país convertida para hectares produtivos de terra/água).

O significado da equação acima, denota a medida de intensidade da pegada ecológica, e esta será tanto maior "quanto: i) maior for o consumo médio por pessoa do país; ii) menor for a eficiência da gestão dos rejeitos desse consumo e; iii) maior for o fator de equivalência, que é uma medida do padrão de consumo do país convertida para hectares produtivos de terra/água" (OLIVEIRA, 2013). Ou seja, quanto mais ecologicamente insustentável for o padrão de desenvolvimento, maior será o valor da pegada ecológica $\left(E F_{P}\right)$

A Pegada Ecológica concede um número dado em hectares produtivos globais, que significa que cidadãos "médios" necessitam de determinada quantidade de hectares produtivos 'médios' de terra e água por ano para satisfazerem suas necessidades e manterem seu padrão de consumo, seu resultado final é um valor de alto apelo intuitivo, pois é evidente que quanto maior a $\mathrm{PE}$, mais ambientalmente comprometedor é o padrão de desenvolvimento de um país, possibilitando perceber que a pressão exercida pelos países de renda elevada é maior do que a exercida pelos de menores níveis ou médio, penalizando países de renda elevada, com um futuro provável comprometedor, com perdas para o meio ambiente (OLIVEIRA, 2013).

O desenvolvimento de novas formas de mensurar a dimensão ambiental, é em geral alvo de críticas devido a confiabilidade dos dados e por corresponderem a índices complexos e que congregam vários indicadores. Contudo, tais intenções possibilitam uma conciliação que é imprescindível para a vertente do desenvolvimento com caráter multidimensional, assim como a viabilidade de bons instrumentos de comparação entre as localidades, que podem sofrer adaptações para melhores performances (ARAÚJO, 2009; BARBOSA, 2009; ROSSATO, 2006).

\section{O IDHA e IDHE como medidas do desenvolvimento sustentável}

O desenvolvimento em sua perspectiva sustentável, inicia-se a partir das preocupações com as condições ambientais, já que o impacto antrópico ao meio ambiente afeta diretamente a qualidade de vida das sociedades, provocando um efeito de causa e consequência. Dessa maneira, o intuito do trabalho é incorporar essa preocupação contemporânea a um índice já consagrado na literatura, o Índice de Desenvolvimento Humano Municipal (IDHM), que foi publicado em 1998 no Brasil, pelo Programa das Nações Unidas para o Desenvolvimento (PNUD) em conjunto com o Instituto de Pesquisa Econômica Aplicada (IPEA) 
e a Fundação João Pinheiro (FJP), visando contribuir para a avaliação de políticas públicas nos municípios e analisar o bem estar dos municípios em termos das principais dimensões do desenvolvimento.

O IDHA e o IDHE agregam as mesmas dimensões tradicionais do desenvolvimento, observadas no IDH, padrão de vida, vida longa e saudável e acesso ao conhecimento, somados a qualidade ambiental e os aspectos biofísicos, que refletem justamente o entendimento sobre o desenvolvimento sustentável, possibilitando verificar o estado ou situação de cada localidade em termos ambientais e biofísicos, e analisar qual o padrão de crescimento que cada localidade vem adotando.

O primeiro passo consiste na criação de um subíndice que representa a dimensão ambiental, em seguida, esse subíndice será incluído no cálculo do IDHM tradicional, obtendo assim o que será chamado de Índice de Desenvolvimento Humano Ambiental (IDHA), ou seja, um indicador com quatro dimensões e; posteriormente, será calculado o IDHE (Índice de Desenvolvimento Humano Ecológico), formado pelo IDH tradicional com a inclusão da Pegada Ecológica. O intuito daqueles indicadores com a dimensão ambiental é de verificar de duas formas distintas, qual(is) o(s) impacto(s) ambientais com relação ao desenvolvimento das localidade, de forma a suprir a inconsistência do IDH, no que tange a inclusão da variável ambiental (sustentabilidade) na estrutura de um indicador consagrado. Ou seja, depois de construído as medidas ambientais - IQA e PIB/EF, segue-se com a inclusão destas ao tradicional IDH.

\section{A Construção do IDHA}

Após o cálculo do IQA, o qual será a quarta dimensão (ambiental) a ser incorporado no IDHM tradicional, este passará a ser chamado de IDHA (Índice de Desenvolvimento Humano Ambiental), conforme apresentado no quadro 2, denominado 'Índice de Desenvolvimento Humano Ambiental (IDHA), trazendo 4 campos em seu bojo.

Quadro 2: Índice Desenvolvimento Humano Ambiental (IDHA).

\begin{tabular}{|c|c|c|c|}
\hline Dimensão Expectativa de vida & Dimensão Conhecimento & Dimensão Padrão de vida & Dimensão Ambiental \\
\hline IDHM Longevidade & IDHM Educação & IDHM Renda & Índice de Qualidade Ambiental (IQA) \\
\hline
\end{tabular}

Fonte: Silva et al. (2004).

Considerando as ponderações metodológicas desenvolvidas aos subíndices que compõem o IDH tradicional publicado pelas Nações Unidas em seus relatórios (RDH), no cálculo do Índice de Desenvolvimento Humano Ambiental (IDHA), realiza-se o ajustamento dos pesos das dimensões, ponderando-os a fim de enquadrar a quarta dimensão (ambiental) naquele indicador, sem deixar de considerar a sua equivalência de índice, conforme adotado por Silva et al. (2004), apresentando como fórmula de cálculo a equação $I D H A=$ $\sqrt[4]{I E x I E V x I P I B x I Q A}$.

Ressalta-se que, a subdimensão ambiental - o IQA, permite, por si só elaborar um ranking para as localidades, que avalie apenas a vertente ambiental, ao passo que o ranking do IDHA agrega as duas perspectivas do desenvolvimento - humano (sustentado) e ambiental (sustentável), examinando se o componente ambiental 'penaliza' o nível de desenvolvimento, de modo a verificar se as localidade 'perdem' 
posições com a inclusão da vertente ambiental no indicador desenvolvimento humano tradicional, assumindo como referência os valores do próprio IDHM tradicional elaborado pelo PNUD/FJP/IPEA.

Portanto, o IPIB é o índice da renda per capita, o IE o índice da educação; o IEV o índice da esperança de vida e; o IQA o índice de qualidade ambiental. O somatório das quatros dimensões (considerando a autoponderação) resulta do IDHA, que tem como proposito equacionar a difícil relação existente entre o desenvolvimento e o ambiente, avançando com a hipótese de que este seria um fator de incremento daquele. Já no caso do IDHE a possibilidade que parece mais intuitiva, nesse raciocínio da inclusão da variável ambiental em um único indicador, seria incluir a pegada ecológica como uma dimensão a mais no IDH, de maneira análoga ao que fizeram Oliveira (2013) e Martins et al. (2006) para o Índice de Sustentabilidade Ambiental.

O IDHE procura ponderar a renda pela Pegada Ecológica, exatamente para penalizar os países que possuem uma alta pegada (mesmo que possuam alto desenvolvimento humano). A ideia é mostrar quais países conseguem um balanço adequado entre acesso ao conhecimento, à saúde, e a um padrão de vida decente e que mantém tais condições para o futuro. Trata-se não de uma medida de eficiência cujo significado seria ranquear países de acordo com sua capacidade de utilizar recursos da forma mais ambientalmente eficiente, mas trata-se de uma medida absoluta de pressão sobre o meio ambiente. Mesmo que possua alto desenvolvimento humano, o país com alta pegada ecológica é entendido como não sustentável e deve, por isso, ser penalizado pelo índice (OLIVEIRA, 2013).

Nesse contexto, de construção de uma métrica ambiental para analisar conjuntamente com as outras dimensões componentes do IDHM dos índices envolve ainda a complicação adicional de tornar comparáveis dados de diferentes fontes, produzidos a partir de escalas distintas, com cobertura e distribuição espacial e temporal diversas, levando à busca de formas alternativas e aproximadas para imputar dados faltantes e construir proxies adequadas e representativas de informações inexistentes.

\section{A Construção do IDHE}

As dimensões do IDH tradicional são mantidas e a metodologia de cálculo sofrerá pequenas modificações. No que tange às dimensões saúde e educação, não há nenhuma modificação. A dimensão 'padrão de vida decente' do IDH é calculada a partir da Renda Nacional Bruta per capita dos países, considerando a paridade de poder de compra (PPC) em dólares de 2005. Tendo em vista que a disparidade de renda entre os países é muito grande, é conveniente a transformação logarítmica da renda, que é o que o PNUD faz antes de normalizar a variável, o que é feito da seguinte forma (denominando a variável utilizada por $Y$ e sendo "i" o país analisado): $I D H_{-} R e n d a_{i}=\frac{\ln (Y i)-\ln (Y \min )}{\ln (Y \max )-\ln (Y \min )}$.

A transformação que se fará busca gerar um indicador que pondere essa dimensão da renda pela Pegada Ecológica. Poderia se pensar em dividir a renda pela Pegada Ecológica, o que equivale matematicamente a utilizar o logaritmo da Pegada, de modo a manter ambas variáveis na mesma escala. 
Logo, a nova dimensão renda pode ser apresentada subtraída da (e não dividida pela) Pegada Ecológica (EF): $I D H_{-} R_{\text {enda }} / E F_{i}=\frac{[\ln (Y i)-\ln (E F i)]-[\ln (Y)-\ln (E F)] \min }{[\ln (Y i)-\ln (E F i)] \max -[\ln (Y)-\ln (E F)] \min }$.

Assim, teremos um indicador da Renda do país descontada da sua pressão sobre o meio ambiente. Fica claro que quanto maior a Pegada Ecológica, menor será o indicador de renda modificada e, portanto, menor será o valor final do seu indicador (que será apresentado aqui de IDHE = Índice de Desenvolvimento Humano Ecológico), que agrega, assim como o IDH, as três dimensões (educação, saúde e renda modificada) por meio de uma média geométrica, como segue: IDHE = $\sqrt[3]{I D H e d u c a c ̧ a o_{i} . I D H l o n g e v i d a d e . I D H \_R e n d a_{i} / E F}$. Essa é a ideia geral do índice, e vale ressaltar que, uma questão importante que se levanta para o indicador do IDHE é que ele possa de fato penalizar as localidades (municípios, estados, regiões e países) de alta Pegada Ecológica e que isso não possa ser compensado por um aumento nas demais variáveis.

\section{DISCUSSÃO TEÓRICA}

Durante muito tempo o crescimento econômico foi utilizado como sinônimo de desenvolvimento na qual a ênfase era dada na produção econômica, simplificado pela necessidade de mensuração que foi dada pela evolução de indicadores como o Produto Interno Bruto (PIB) e o PIB per capita, devido à falta de um indicador adequado para mensurar o bem-estar, progresso ou qualidade de vida. É inegável a importância que tais indicadores revelam ao apresentarem quantificados os níveis de atividades econômicas de uma localidade, porém a insuficiência de tais variáveis para responder as questões ampliadas que envolvem o processo complexo do desenvolvimento são evidentes, pois nem sempre elevações nesses indicadores refletem em maiores acesso da população a saúde, educação, riqueza material, cultural dentre outros, não demonstrando de fato, se o nível de vida da população melhorou ou não.

Por mais que para muitos os termos crescimento e desenvolvimento econômico aparentem ser sinônimos, os vocábulos são distintos, não apenas conceitualmente, mas, sobretudo em seu propósito, já que os objetivos do desenvolvimento ultrapassam a riqueza material, sendo que o crescimento é indispensável, porém não suficiente para se alcançar conquistas sociais e melhorias na qualidade de vida. Dessa maneira, o crescimento econômico é qualificado como instrumental que pode estimular o desenvolvimento, mas não efetivamente garantir, já que este possui objetivos que vão além do simples aumento do PIB, inclusive esse aumento pode ser acompanhado por mazelas como: pobreza, desigualdade e não ampliação emprego, ocasionando externalidades negativas para a sociedade e sendo considerado como mau desenvolvimento (SACHS, 2008).

Em 1990, ocorreu à publicação do Relatório do Desenvolvimento Humano (RDH) pelo Programa das Nações Unidas para o Desenvolvimento (PNUD), que buscou findar a ideia que imperava até a década de 70, no qual o desenvolvimento era sinônimo de progresso material (crescimento). Então, o economista paquistanês Mahbud ul Haq juntamente com a colaboração de Amartya Sen e seu pensamento, criaram um 
indicador sintético com o objetivo de formar um hodômetro do desenvolvimento de um país, já que avaliação e aferição desse processo era uma dificuldade a ser solucionada (SACHS, 2008).

Conhecido como Índice de Desenvolvimento Humano (IDH), de fácil mensuração e simplista, sendo um método alternativo às medidas baseadas tão somente em aspectos econômicos, como o PIB e o PIB per capita, incluindo novas dimensões como de expectativa de vida e acesso ao conhecimento que enriqueceram a noção de desenvolvimento, expondo um novo modo de se entender o desenvolvimento, tornando-se um indicador razoável para este fim (VEIGA, 2010).

No interior das discussões sobre o desenvolvimento foram incorporados novos requisitos para alcançar tal concepção, de modo harmônico e integral, como a inclusão de novos desafios e até mesmo dimensões pouco exploradas pelas teorias tradicionais do desenvolvimento, assumindo diferentes nomenclaturas. Nesse contexto, destaca-se a sustentabilidade, com propósito de utilizar os recursos naturais de maneira prudente, particularmente em economias de base produtiva assentadas no uso intensivo de recursos naturais, possibilitando uma conciliação que é imprescindível para a vertente do desenvolvimento com caráter multidimensional, assim como a viabilidade de bons instrumentos de comparação entre as localidades, que podem sofrer adaptações para melhores performances.

Em 1982, criou-se a Comissão Mundial sobre o Meio Ambiente e Desenvolvimento, na qual os resultados vieram a públicos em 1987, em um documento conhecido como Nosso futuro comum, também chamado de Relatório Brundtland (1991), em que propunha cautela e preocupação com o risco ambiental do crescimento econômico expressando o entendimento como desenvolvimento sustentável (ROMEIRO, 2012).

O fator ambiental exerce grande influência na qualidade de vida dos indivíduos, sendo por influência em questões como a saúde, habitacionais, saneamento básico, lazer dentre outros, principalmente em localidades que detém grandes riquezas naturais e vasta biodiversidade. Entretanto, essa diversidade pode ser comprometida devido às pressões antrópicas, causando sérios problemas ambientais com consequências sociais, como a falta de saneamento básico, destinação inadequada para o lixo, e péssimas condições de habitação para as populações (ARAÚJO, 2009).

Desse modo, a utilização de indicadores que retratem a 'situação da sustentabilidade', de forma simples, que defina a própria ideia é notoriamente importante. Sendo que o processo de desenvolvimento destes indicadores deve contribuir para uma melhor compreensão do que seja o desenvolvimento sustentável, sempre focando as mudanças nas tendências mundiais que hoje podem ser sintetizadas como sendo: a necessidade de uma visão mais dinâmica que envolve riscos globais; problemas de crescimento populacional e de urbanização; aguçamento da dualidade socioeconômica; rupturas e descontinuidades tecnológicas; consciência intergeracional (FAUCHEUX et al., 1995). Na perspectiva de sustentabilidade, o escopo é caracterizar a interação entre a atividade antrópica e o meio ambiente, bem como trabalhar um conceito que é complexo, dinâmico e diverso.

Desta forma, o maior desafio da sustentabilidade, é a quantificação, de modo a efetuar indicadores ambientais para produzir um retrato, ou um conjunto de sinais que facilitam a avaliação do progresso de 
uma determinada região na busca pelo desenvolvimento sustentável, sendo ferramentas crucias no processo de identificação de problemas, reconhecimento dos mesmos, formulação, implementação e avaliação de políticas. Nesse sentido, a nova dimensão refere-se à sustentabilidade ambiental, acrescida à sustentabilidade social, fundamenta-se em uma solidariedade tanto sincrônica quanto diacrônica com as gerações atuais e futuras, respectivamente.

As ações antrópicas causam impacto ao meio ambiente e o impacto do meio ambiente sobre a qualidade de vida, principalmente em consequências do desenvolvimento acelerado, sem muitas vezes um controle sobre as condições do meio ambiente, que compromete a vida e a saúde dos seres vivos (ARAúJO, 2009).

\section{Revisão dos procedimentos metodológicos de construção do 'IQA' e alguns resultados empíricos do IDHA}

Na literatura, em particular na econômica, não existe definição clara sobre à utilização de variáveis ambientais exatas para expressarem a qualidade ambiental, sendo determinado pelos critérios e objetivos do próprio pesquisador, por meio de variáveis que possibilitem tal propósito, utilizando informações estatísticas disponíveis e dentro da realidade da localidade, bem como o motivo do ano de referência de informações utilizadas serem distintas, devido a indisponibilidade de série de dados para o mesmo ano (BARBOSA, 2009). A pesquisa realizada por Rossato (2006), teve como objetivo construir uma dimensão ambiental, também denominada de Índice de Qualidade Ambiental (IQA) para o estado do Rio Grande do Sul, agregando as condições socioeconômicas, pelas dimensões tradicionais do desenvolvimento humano para compor o denominado Índice de Desenvolvimento Humano Ambiental IDHA para o estado.

Os resultados do estudo mostraram que o Índice de Qualidade Ambiental para o estado do Rio Grande do Sul possui um IQA médio de 0,55 , significando que a qualidade ambiental está 45 pontos percentuais abaixo do máximo de $100 \%$, enquanto o IDH Ambiental construído e proposto para indicar o nível de desenvolvimento apresentou um valor médio foi de 0,25 . Portanto, ao se considerar a qualidade ambiental o índice de desenvolvimento humano decresceu, o que mostra que a dimensão ambiental também é importante na construção do IDH, e principalmente para alcançar um desenvolvimento considerado sustentável, portanto, a inclusão do nível de qualidade ambiental do estado constituiu um redutor do nível de desenvolvimento humano.

Nesse contexto, Barbosa (2009) investigou a qualidade do ambiente e de vida nos municípios do estado de Minas Gerais, por meio da quantificação de tais dimensões, com a elaboração de uma dimensão ambiental, o IQA seguido da construção de um Índice de Desenvolvimento Humano Ambiental, utilizando os mesmo procedimentos metodológicos desenvolvidos por Rossato (2006), por meio da análise multivariada, com base na análise fatorial por componentes principais, identificando o nível de desenvolvimento humano do estado, e incorporando, na análise, o meio ambiente. Os resultados mostram o Índice de Qualidade Ambiental para o estado de Minas Gerais possui um IQA médio de 0,51, significando que a qualidade ambiental está 49 pontos percentuais abaixo do máximo de 100\%, para o IDH Ambiental o valor médio foi de 0,42 . Portanto, ao se considerar a qualidade ambiental o índice de desenvolvimento humano decresceu. 
Araújo (2009), com o mesmo intuito de verificar as condições ambientais, realizou um estudo para o estado da Bahia, criando o Índice de Condições Ambientais - ICA, através da técnica de análise fatorial, desse modo, o Estado da Bahia apresentou um ICA médio de 0,34 , indicando o baixo nível das condições ambientais do estado, assim como o Índice de Desenvolvimento Socioambiental construído apresentou uma média de 0,28. Em seguida, o ICA foi agregado aos indicadores que compõe o IDH (dando ao mesmo uma abordagem ambiental), criando o Índice de Desenvolvimento Socioambiental - IDSA, apresentou uma média de 0,28, o que reduz o nível de desenvolvimento humano.

Portanto, os três estudos (ROSSATO, 2006; ARAÚJO, 2009; BARBOSA, 2009) realizados constataram que ao se considerar as condições ambientais dos Estados, o nível de desenvolvimento humano reduz, ou seja, a condição do meio ambiente se constitui um fator que reduz o processo de desenvolvimento, especialmente o sustentável. Essas análises ambientais possibilitam verificar a influência do ambiente na qualidade de vida das populações, já que existe uma relação direta entre tais elementos. Além de proporcionar um alerta aos riscos que a população pode sofrer, principalmente, no que se refere as condições dos serviços sociais, uso prudente de recursos naturais, tais como água, floresta e um ambiente com boas condições sanitárias.

\section{Revisão dos procedimentos metodológicos de construção da 'EF' e alguns resultados empíricos do IDHE}

A Pegada Ecológica é um dos indicadores de sustentabilidade que considera aspectos ecológicos e não levam em conta as dimensões tradicionais do Desenvolvimento como social, econômica e institucional. A integração da Pegada ecológica aos componentes tradicionais do Índice de Desenvolvimento Humano, foi uma proposta de Oliveira (2013), com o objetivo de atribuir um 'peso ecológico', a partir da Pegada Ecológica, capaz de mensurar o impacto ambiental (via recursos e degradação) gerado por uma determinada localidade. O resultado deve mostrar um novo indicador que penaliza localidades com alto impacto ambiental, contribuindo para formulação de informações essências para o debate acadêmico e para formulação de políticas públicas.

Essa metodologia é inovadora, já que considera os aspectos biofísicos aos tradicionais componentes do desenvolvimento, dessa forma, poucos estudos foram realizados nesse sentido, utilizando tal agregação. O trabalho realizado por Oliveira (2013), utilizou dados do IDH e da Pegada Ecológica para os países, gerando um novo indicador denominado Índice de Desenvolvimento Humano Verde, o IDHV, e dividiu em grupos para facilitar a análise como: Muito Elevado >0,75; Elevado, entre 0,65 e 0,75; Médio, entre 0,5 e 0,65; e Baixo < 0,5 .

Os resultados mostraram que esse índice apresentou um ranking diferenciado, privilegiando os países com alto desenvolvimento humano e Pegada Ecológica mais baixa, como por exemplo a Coréia do Sul, apresentando a primeira colocação, pois possui o 120 IDH e uma pegada de apenas 1,3. Países que aparecem bem no IDHV, possuem alto desenvolvimento humano e relativamente baixa Pegada, enquanto que países com alta pegada ecológica foram penalizados pelo índice. 


\section{CONCLUSÕES}

A preocupação com o meio ambiente já não é apenas uma questão das ciências ambientais; há tempos, transformou-se em um problema interdisciplinar. Nesse sentido a interação de aspectos econômicos, sociais apresentam-se de forma relevante para reduzir os problemas ambientais, visto que o problema ecológico resulta de ações econômicas assim como das desigualdades sociais, deste modo às ações devem ser sustentáveis, levando em conta as diversas dimensões desenvolvimento.

Tais indicadores possibilitam mostrar a interface sustentável de uma determinada localidade, a realização deste estudo procura preencher a lacuna existente no que se refere à necessidade de incorporar a dimensão ambiental, juntamente, as dimensões consideradas tradicionais do indicador de desenvolvimento, de modo que a aquela dimensão reflita o nível de sustentabilidade. Além disso, buscou-se relacionar a qualidade ambiental e a qualidade de vida das população por meio da inclusão do Índice de Qualidade Ambiental e Pegada Ecológica no Índice de Desenvolvimento Humano, já que o impacto causado pelo homem ao meio ambiente e o impacto do meio ambiente sobre a qualidade de vida apresenta uma relação direta, e muitas vezes de grande complexidade.

Araújo (2009), destaca “[...] a destruição das florestas pela prática intensa de desmatamento e queimadas, a chuva ácida e o buraco na camada de ozônio e o efeito estufa refletem as consequências do desenvolvimento acelerado, sem controle da qualidade ambiental". Desse modo, as condições do meio ambiente influenciam diretamente o nível de desenvolvimento de uma população, principalmente no tocante ao desenvolvimento considerado sustentável, a integração das três dimensões - socioeconômica e ambiental - busca suprir as deficiências de ambos por meio de sua complementariedade, parecendo ser uma alternativa lógica plausível, através dos novos indicadores IDHA e IDHE, penalizando localidades que conseguem um alto desenvolvimento humano às custas do meio ambiente e beneficiando aqueles que $o$ conseguem sem grandes danos.

Tal proposta contribui para a construção de uma ferramenta prática de comparação, servindo como esclarecedora na relação entre homem e o meio ambiente e, além disso, permiti construir análises econométricas que verificam o impacto por intermédio de regressões, a saber qual dimensão mais e menos contribui para alcançar o desenvolvimento sustentável. A construção desses indicadores é uma imprescindível ferramenta na tomada de decisão no campo das políticas públicas, e possibilita a comparação entre localidades, favorecendo um aprimoramento técnico-metodológico.

\section{REFERÊNCIAS}

ARAÚJO, L. V.. Elaboração de um índice de desenvolvimento socioambiental para o Estado da Bahia. Dissertação (Mestrado em Economia Aplicada) Universidade Federal de Viçosa, Viçosa, 2009.

BARBOSA, F. F. B.. Índice de qualidade socioambiental para o Estado de Minas Gerais. Dissertação (Mestrado em Economia) - Universidade Federal de Viçosa, Viçosa, 2009.
CERVI, J. L.; CARVALHO, P. G. M.. A Pegada Ecológica: breve panorama do estado das artes do indicador de sustentabilidade no Brasil. In: Encontro Nacional da Sociedade Brasileira de Economia Ecológica, 7. Anais. Fortaleza: 2007.

ENRÍQUEZ, M. A.. Trajetórias do desenvolvimento: da ilusão do crescimento ao imperativo da sustentabilidade. Rio de Janeiro: Garamond, 2010. 
FAUCHEUX, S.; NOËL, J. F.. Economia dos recursos naturais e do meio ambiente. Lisboa: Instituto Piaget, 1995

FEITOSA, M. J. S.; GOMÉZ, C. R. P.; CÂNDIDO, G. A.. Pegada ecológica municipal: uma análise da (in)sustentabilidade ambiental dos municípios de João Pessoa e Campina Grande. Revista Metropolitana de Sustentabilidade, São Paulo, v.3, n.3, p.49-65, 2013

FIRMINO, A. M.; SANTOS, H. N.; PINA, J. H. A.; RODRIGUES, P. O.; FEHR, M.. A relação da pegada ecológica com o desenvolvimento sustentável/cálculo da pegada ecológica de Toribaté. Caminhos de Geografia, Uberlândia, v.10, n.32, p.41-56, 2009.

FONSECA, J. J. S.. Metodologia da pesquisa científica. Fortaleza: UEC, 2002

GALLOPÍN, G.. Asystems approach to sustainability and sustainable development, Santiago de Chile, v.1, n.64, 2003.

GALLOPÍN, G.. Environmental and sustainability indicators and the concept of situational indicators: a systems approach. Environmental Modeling and Assessment, v.1, n.3, p.101-117, 1996.

GUIMARÃES, R. P.. Aterrizando una Cometa: indicadores territoriales de sustentabilidad. Serie Investigación, Santiago do Chile, v.18, n.98, 1998

GUIMARÃES, R. P.; FEICHAS, S. A. Q.. Desafios na construção de indicadores de sustentabilidade. Ambiente e Sociedade, São Paulo, v.12, n.2, 2009.

LISBOA, C. K.; BARROS, M. V. F.. A pegada ecológica como instrumento de avaliação ambiental para a cidade de Londrina. Confins, São Paulo, v.1, n.8, 2010.

MARTINS, A. R. P.; FERRAZ, F. T.; COSTA, M. M.. Sustentabilidade Ambiental como Nova Dimensão do Índice de Desenvolvimento Humano dos Países. Revista do BNDES, Rio de Janeiro, v.13, n.26, p.139-162, 2006.

MIKHAILOVA, I.. Sustentabilidade: Evolução dos conceitos teóricos e os problemas da mensuração prática. Revista Economia e Desenvolvimento, Rio Grande do Sul, v.1, n.16, p.22-41, 2004. DOI: http://doi.org/10.5902/red.v0i16.3442

MORAES, O. J.. Economia ambiental: instrumentos econômicos para o desenvolvimento sustentável. São Paulo: Centauro, 2009.

MOURA, M. E.; SANTOS, M. E.; JESUS, T. S.; SOUZA, R. M. Desenvolvimento de indicadores de sustentabilidade urbano-regional. In: ENCONTRO DE GÉOGRAFOS DA AMÉRICA LATINA, 10. Anais. São Paulo: USP, 2005.

OLIVEIRA, W. F.. Índice de Desenvolvimento Humano e Pegada Ecológica: uma proposta de integração. In:
ENCONTRO REGIONAL DE ECONOMIA DA REGIÃO SUL, 16 Anais. Porto Alegre: ANPEC Sul, 2013.

QUIROGA, R.. Indicadores de sostentabilidad y desarrollo sostenible: estado del arte y

perspectivas. Santiago de Chile: DMAAH, 2001

ROMEIRO, A. R.. Desenvolvimento sustentável: uma perspectiva econômico ecológica. Estudos avançados, São Paulo, v.26, n.74, p.65-92, 2012. DOI: http://doi.org/10.1590/S0103-40142012000100006

ROSSATO, M. V.. Qualidade ambiental e qualidade de vida nos municípios do estado do Rio Grande do Sul. Tese (Doutorado em Economia Aplicada) - Universidade Federal de Viçosa, Viçosa, 2006.

SACHS, I.. Desenvolvimento: Includente, Sustentável, Sustentado. Rio de Janeiro: Garamond, 2008.

SANDRONI, P.. Dicionário de Economia. 4 ed. São Paulo: Record, 2007.

SICHE, R.; AGOSTINHO, F.; ORTEGA, E.; ROMEIRO, A.. Índices versus indicadores: precisões conceituais na discussão da sustentabilidade de países. Ambiente e Sociedade, São Paulo, v.10, n.2, p.137-148, 2007. DOI: http://doi.org/10.1590/S1414-753X2007000200009

SILVA, A. C. G.; FERREIRA, R. P.. Monografia (Graduação em Economia do Meio Ambiente) - Universidade do Porto, Lisboa, 2004

SILVEIRA, D. T.; CÓRDOVA, F. P.. A pesquisa científica. In GERHARDT, T. E.; SILVEIRA, D. T.. Métodos de Pesquisa. Porto Alegre: EDUFRGS, 2009. p.31-42.

SOARES, A. C. L. G.; GOSSON, A. M. P. M.; MADEIRA, M. A. L. H.; TEIXEIRA, V. D. S.. Índice de Desenvolvimento Municipal: Hierarquização dos Municípios do Ceará no ano de 1997. Paraná Desenvolvimento, v.1, n.97, p.71 -89, 1999.

SOLIGO, V.. Indicadores: conceito e complexidade do mensurar em estudos de fenômenos sociais. Estudos em Avaliação Educacional, v.23, p.12-25, 2012

SOUZA, A. C.. Sustentabilidade: a busca por mensuração através de indicadores. In: ENCONTRO NACIONAL DA ECOECO, 11. Anais. São Paulo: 2004.

TRZESNIAK, P.. Indicadores quantitativos: reflexões que antecedem seu estabelecimento. Ciência da Informação, Brasília, v.27, n.2, p.159-164, 1998.

VEIGA, J. E.. Desenvolvimento sustentável: o desafio do século XXI. Rio de Janeiro: Garamond, 2010.

A CBPC - Companhia Brasileira de Produção Científica (CNPJ: 11.221.422/0001-03) detém os direitos materiais desta publicação. Os direitos referem-se à publicação do trabalho em qualquer parte do mundo, incluindo os direitos às renovações, expansões e disseminações da contribuição, bem como outros direitos subsidiários. Todos os trabalhos publicados eletronicamente poderão posteriormente ser publicados em coletâneas impressas sob coordenação da Sustenere Publishing, da Companhia Brasileira de Produção Científica e seus parceiros autorizados. Os (as) autores (as) preservam os direitos autorais, mas não têm permissão para a publicação da contribuição em outro meio, impresso ou digital, em português ou em traduçăo. 